\title{
Checkpoint inhibitors: literature review of new treatments for hepatocellular carcinoma
}

\author{
Layana Biglow $^{1}$, Sara Ashraf ${ }^{2}$, Mohamed Alsharedi ${ }^{2}$ \\ ${ }^{1}$ Department of Internal Medicine, Joan C. Edwards School of Medicine, Marshall University, Huntington, WV, USA; ${ }^{2}$ Division of Hematology \\ and Oncology, Joan C. Edwards School of Medicine, Marshall University, Edwards Comprehensive Cancer Center at Cabell Huntington Hospital, \\ Huntington, WV, USA \\ Contributions: (I) Conception and design: M Alsharedi; (II) Administrative support: L Biglow, S Ashraf; (III) Provision of study materials or patients: \\ None; (IV) Collection and assembly of data: L Biglow, S Ashraf; (V) Data analysis and interpretation: None; (VI) Manuscript writing: All authors; (VII) \\ Final approval of manuscript: All authors. \\ Correspondence to: Layana Biglow. Department of Internal Medicine, Joan C. Edwards School of Medicine, Marshall University, Huntington, WV, \\ 25701, USA. Email: biglow@marshall.edu.
}

\begin{abstract}
Objective: To systematically review the ongoing progress of effective treatment of advanced hepatocellular carcinoma (HCC), mainly focusing on immune checkpoint inhibitors (ICPI) as monotherapy and combination therapy.

Background: HCC in general has a poor prognosis; particularly in the advanced stage. For more than 10 years, the treatment with multikinase inhibitors was the first line treatment. Before the introduction of checkpoint inhibitors, very few treatments were available for patients with hepatocellular cancer in the advanced stage, especially in metastatic and unresectable disease.

Methods: We performed an extensive search of the ongoing and published clinical trials in the English written literature concerning of HCC with immune checkpoint inhibition when compared to first line chemotherapy.

Conclusions: The treatment paradigm for advanced stage HCC has significantly changed recently with the introduction of immunotherapy; based on existing research, there is new era for HCC treatment which will positively affect the outcome in a malignancy that did not see therapy advancement for more than a decade. Monoclonal antibodies against programmed death ligand 1 (PD-L1) and programmed cell death protein 1 (PD-1), such as nivolumab and pembrolizumab appear to be a promising therapeutic option in HCC. This review outlines immunotherapy that has been approved, and what inhibitors are under investigation for patients with advanced stage HCC.
\end{abstract}

Keywords: Immunotherapy; immune checkpoint inhibitor (ICPI); hepatocellular carcinoma (HCC); metastatic disease

Received: 09 May 2021; Accepted: 22 September 2021; Published: 10 November 2021.

doi: 10.21037/sci-2021-029

View this article at: https://dx.doi.org/10.21037/sci-2021-029

\section{Introduction}

Liver and intrahepatic bile duct cancer accounts for $2.4 \%$ of all new cancer cases in 2019 and is responsible for $5.2 \%$ of all cancer deaths in the US (1). In 2016, there were approximately 83,000 people living with liver and intrahepatic duct carcinoma and according to most recent data, 5 -year survival of these patients is at $18.4 \%$ (1). Hepatocellular carcinoma (HCC) is the most common primary malignancy of the liver, which is the sixth most common cause of cancer and ranks fourth among the causes of cancer related deaths (2). HCC has a plethora of risk factors, including viral hepatitis, alcohol abuse and the two most common risk factors in the US: nonfatty liver disease 
and nonalcoholic steatohepatitis (3). Hepatitis B virus (HBV) is the most common cause for HCC globally, and chronic infection increases the relative risk for developing HCC 15-20 times with a mortality rate of about $40 \%$ for all cases of the disease. In endemic countries, $\mathrm{HBV}$ is transmitted perinatal or vertical transmission as compared to nonendemic countries where transmission is usually from needle sharing and sexual contact amongst intravenous drug users (3). Chronic liver damage from viral hepatitis triggers fibrogenesis, leading to cirrhosis. The presence of cirrhosis and $\mathrm{HBV}$ infection simultaneously further increases the risk of developing HCC (3). Over $80 \%$ of HCC patients present with symptoms at advance stages such as Barcelona Clinic Liver Cancer (BCLC) Stage C or Stage D, when local and/ or curative agents such as tumor resection, ablation or trans arterial chemoembolization or trans-arterial chemoablation (TACE), are no longer viable choices and 5-year survival rates just $18 \%$ (4). There was no effective treatment until 2008 when sorafenib was approved by FDA as a front-line agent based on phase III SHARP trial in which sorafenib demonstrated improvement of survival compared to best supportive care alone (5).

Subsequently, in the REFLECT study, lenvatinib has demonstrated noninferiority to first line sorafenib in patient with unresectable HCC and no prior systemic therapy (6).

Sorafenib is an anti-tyrosine kinase inhibitor of vascular endothelial growth factor (VEGF), the integral rate limiting step of angiogenesis, and is first line for advanced HCC, while lenvatinib, an oral TKI which inhibits multiple factors including VEGF receptors 1-3, fibroblast growth factor receptors (FGFR 1-4), platelet-derived growth factor receptor (PDGFR)- $\alpha$, RET and KIT, was shown to be noninferior to sorafenib in terms of overall survival (OS), but had higher grade (3 and above) treatment related-emergent adverse effects (4). Despite being first and second-line treatments, the objective response rates (ORR), and OS of theses multikinase inhibitors are limited. Patient ability to tolerate was generally low, indicating the urgent need for more tolerable, and more effective treatment options.

Over a decade, there was not much advancement in the treatment of HCC, until recently with addition of immunotherapy in solid tumors. Immune checkpoint inhibitors (ICPI) were a natural area of exploration for advanced HCC due to benefits seen in other malignances, such as melanoma, renal cell carcinoma, triple negative breast cancer and non-small cell lung cancer. This review focuses on the progress made with ICPI as monotherapy and in some form of combination therapy for the treatment of advanced HCC. It highlights the impressive combination of atezolizumab, an ICPI with a VEGF inhibitor, which has finally after a decade, surpassed sorafenib in the treatment of advanced HCC.

We present the following article in accordance with the Narrative Review reporting checklist (available at https:// dx.doi.org/10.21037/sci-2021-029).

\section{Immunotherapy}

Sorafenib has been the only first line therapy with a significant OS for advanced HCC for more than ten years until very recently, when Finn and colleagues brought light to the successful combination of atezolizumab and bevacizumab for the treatment of advanced HCC in the New England Journal of Medicine. Prior to this, no other treatment exceeded sorafenib, leading to longer OS and progression free survival (PFS) that were statistically significant with superior patient reported outcomes (7).

\section{Combination of PD-L1 inhibitor and VEGF inhibitor}

\section{Atezolizumab and bevacizumab}

Atezolizumab is an anti-programmed death ligand 1 (PD-L1) antibody and bevacizumab (Avastin) is an antiVEGF antibody which also has immunoregulatory effect on the immune system's milieu. Preclinical data showed that addition of atezolizumab may have decreased the immunosuppressive cells such as those derived from myeloid tissue and specialized macrophages. It may also be helpful in recruitment of cytotoxic $\mathrm{T}$ cells into the tumor setting (8).

On May 29, 2020, the USFDA approved atezolizumab with bevacizumab for the treatment of people with non-operable or metastatic HCC with chemo- or immunotherapy, based on the momentous IMbrave150 trial.

\section{IMbrave150}

The IMbrave150 was a global, open label randomized trial of atezolizumab plus bevacizumab versus sorafenib as treatment of metastatic or unresectable HCC (7). Five hundred and one patients were randomly assigned to receive either atezolizumab plus bevacizumab or sorafenib. Treatment was continued until it was too toxic or loss of clinical benefit. The study included locally advanced metastatic or unresectable HCC patients with an Eastern 
Cooperative Oncology Group (ECOG) performance status of $0-1$ and Child-Pugh A cirrhosis. They could not have had any previous systemic therapy, but prior locoregional therapy was acceptable.

Patients who carried a diagnosis of autoimmune disease or prior diagnosis of Hepatitis B or Hepatitis C were not included in the study sample. All patients had to undergo EGD within 6 months prior to beginning and untreated or partially treated esophageal or gastric varices or high risk for bleeding were not incorporated. Patients with myocardial infraction or stroke within past 12 months were not eligible either. Characteristics at baseline were well balanced on both arms.

They were organized by geographic area (Asia except Japan $v s$. the rest of the world); Macrovascular invasion or spread of disease outside of the liver (presence or absence); Baseline alpha fetoprotein (AFP) level (<400 or $\geq 400$ ); ECOG 0 or 1.

Atezolizumab-bevacizumab group received $1.2 \mathrm{~g}$ of atezolizumab plus $15 \mathrm{mg}$ per kilogram of body weight of bevacizumab intravenously every 3 weeks. Patients assigned to the sorafenib group received $400 \mathrm{mg}$ of sorafenib orally twice daily.

Dose modifications were not permitted in the atezolizumab +/- bevacizumab group but were allowed in the sorafenib group. Patients who discontinued either monotherapy or combined treatment because of an adverse event were permitted to continue taking single-line treatment as long as there was clinical benefit (8).

Tumors were assessed by computed tomography (CT) or magnetic resonance imaging (MRI) at baseline and every 6 weeks until week 54 and then every 9 weeks thereafter. Safety was constantly evaluated by vital signs, laboratory values and assessment of the incidence and severity of adverse outcomes according to the National Cancer Institute (NCI) Common Terminology Criteria for Adverse Events. Patient-described outcomes were evaluated with the use of the European Organization for Research and Treatment of Cancer quality-of-life questionnaire for cancer (EORTC QLQ-C30).

Multiple primary end points were OS and PFS. Median OS was not accomplished in the patients who received atezolizumab plus bevacizumab and was 13.2 months in the patients who received sorafenib. Median PFS was 6.8 months in the combination arm versus 4.3 months in the sorafenib arm.

Additional end points consisted of ORR; duration of response; time to deterioration of activities of daily living and quality of life reported by the patient on the EORTC QLQ-C30.

The ORR per Response Evaluation Criteria in Solid Tumors (RECIST) 1.1 was $28 \%$ in the atezolizumab plus bevacizumab group compared with $12 \%$ in the sorafenib group. The ORR per modified RECIST was $33 \%$ vs. $13 \%$ respectively.

Combination arm delayed time to deterioration (TTD) in patients were reported as decreased in oral intake, pain, and diarrhea as compared to sorafenib. In terms of grade 3 or 4 adverse outcomes, $25.8 \%$ patients developed at least one adverse event related to atezolizumab, $23 \%$ related to bevacizumab and $30 \%$ related to sorafenib (8).

Significantly better OS and PFS outcomes were seen with atezolizumab + bevacizumab than with sorafenib in patients with unresectable disease who had received no previous systemic treatment. Benefit was generally consistent across clinical subgroups. Serious toxic effects were shown in $38 \%$ of the patients who underwent the combination therapy; however, no unexpected toxic effects were observed. Some of the adverse effects were rash, bleeding/hemorrhage, infusion reaction, diarrhea, hepatitis and pruritis, with hypertension, proteinuria and fatigue being the most common. Combination therapy also resulted in a longer TTD for patient-reported quality of life and performance than sorafenib (9).

Prior to this, there were only two approved ICPIs to treat hepatocellular cancer following disease progression on first line therapy, nivolumab and pembrolizumab. In May 2017, pembrolizumab was approved for microsatellite instability-high (MSI-H) or DNA mismatch repair deficient (dMMR) non-operable, or metastatic solid tumors; again in 2018 pembrolizumab was approved in the second line setting for advanced stage HCC based on the results of KEYNOTE-224. Whereas nivolumab gained the approval for second line setting in 2017. Interestingly, all approvals for ICPIs were independent of PD-L1 tumor or immune cell expression.

In March 2020, the USFDA approved the combination of nivolumab and ipilimumab for treatment of patients with advanced HCC formerly treated with sorafenib (1). Regulatory approval was largely based on results of the phase I/II CheckMate 040 trial, in which nearly 150 sorafenib-treated patients with advanced HCC and no worse than Child-Pugh A cirrhosis were treated with three different schedules of combined therapy (2). In the three groups combined, objective rate was $31 \%$ and complete response rates were $5 \%$, while median response duration 
was 17 months. The rate of grade 3 or 4 treatment-related adverse events was 37 percent, most commonly pruritus and rash. When immunotherapy is selected for treatment of advanced HCC in patients with an adequate performance status, we now generally prefer combined therapy over monotherapy with nivolumab or pembrolizumab.

\section{PD-1 and PD-L1 inhibitors}

ICPI block the negative regulatory signals in 2 ways: by effecting $\mathrm{T}$ cells directly or effecting cells that interact with $\mathrm{T}$ cells, such as tumor cells and APCs, which help stimulate anti-tumor immunity already present in the body (6). Programmed cell death protein 1 (PD-1) is mainly expressed on $\mathrm{CD}^{+} \mathrm{T}$ cells, and by frequent antigen stimulation, IFN-gamma induces IRFP which binds to Pdcd-promoter, jumpstarting PD-1 transcription in T cells. When PD-1 connects with its ligands, PD-L1 or PD-L2, T cell multiplication and cytokine release are hindered (10). PD-L1 is detected in 74\% of HCC cases, along with immune cells, and could possibly be used as a biomarker for recurrence or prognosis of HCC pts after resection (10). Recent studies have showed that PD-1/PD-L1 inhibitor therapy alone is safe and tolerable.

\section{Nivolumab}

Nivolumab, a human IgG4 monoclonal antibody inhibitor of PD-1 receptor, gained conditional approval as the first ICPI treatment for patients with advanced HCC who were previously treated with sorafenib evidenced by the results of the landmark CheckMate 040 trial.

\section{CheckMate 040}

CheckMate 040 trial was a phase I/II, multicenter, noncomparative dose escalation and expansion study that evaluated the safety and efficiency of nivolumab in patients with histologically confirmed advanced $\mathrm{HCC}+/-$ hepatitis $\mathrm{B}$ or $\mathrm{C}$ infection. Patient selected for this trial had ChildPugh scores 7 or less, ECOG performance status 0 or 1, and $\mathrm{HBV}$ on current antiviral treatment with viral load less than $100 \mathrm{IU} / \mathrm{mL}$. In the dose-escalation phase, patients were divided into three cohorts: hepatitis $\mathrm{C}$ virus (HCV) infected, HBV infected and patient without viral hepatitis. Across the cohorts, patients were given nivolumab dosed per kilogram intravenously every 2 weeks with the intent of determining the max tolerated dose. Nivolumab $3 \mathrm{mg} / \mathrm{kg}$ was given every 3 weeks to patients in dose-expansion phase separated into 4 cohorts: sorafenib naïve or intolerant without viral hepatitis, patients who progressed on sorafenib without hepatitis B and C infection. Primary endpoints included safety and ability to tolerate for the escalation phase, and ORR for the expansion phase.

Two-hundred and sixty-two eligible patients were included in the study; 48 and 214 in dose-escalation and dose-expansion phases respectively with overall median age of 62 years, and 37 (77\%) of 48 patients had been previously treated with sorafenib. The objective response rate was $15 \%$ for the escalation phase, the disease CR was $58 \%$, the median time-to-progression was 3.4 months, and the 6- and 9-month OS rates were both approximately $66 \%$. The median OS was 15 months for the escalation phase. Grade 2 impairment was found in one patient without viral hepatitis, and a maximum tolerated dose was unknown. Treatment-related adverse events included rash, increase in aspartate aminotransferase (AST)/alanine aminotransferase (ALT), lipase, amylase and pruritis, with serious TRAE in 3 patients which include pemphigoid, adrenal insufficiency, and liver disorder. Sixty-three percent of subjects died in the escalation phase with causes not related to nivolumab therapy. In dose-expansion phase, disease progression was the most common cause for discontinuation of treatment, occurring in $62 \%$ of patients. The ORR was $20 \%$ in patients who received $3 \mathrm{mg} / \mathrm{kg}$ every 2 weeks in the doseexpansion phase. Overall, it was concluded that nivolumab showed a manageable safety profile with acceptable tolerability and based on ORRs showed promising potential of nivolumab as a treatment for advanced HCC (11).

\section{Finkelmeier et al.: Feasibility and safety of nivolumab}

A retrospective cohort study between three German universities was conducted to retrospectively analyze reallife experience of HCC patients treated with nivolumab. Patients included in this study had confirmed BCLC stage $\mathrm{B}$ and $\mathrm{C}$ disease, Child-Pugh stages A-C, were sorafenib naive or had previous treatments for a median of 14 weeks duration, had undergone surgery or ablation for curative purposes, and those who had TACE or selective internal radiation therapy (SIRT). Through EMR review, survival was tabulated as the time from start of nivolumab therapy until death from any cause or last patient encounter, and the outcome was qualified as a range from stable disease to disease resolution. The median follow-up time was 100 days, and median treatment duration was 8 weeks with median of 4 cycles of nivolumab (range, 1-42 cycles). The primary endpoint was median OS. 
Thirty-four patients were included in this study with male predominance $(76.5 \%)$, with a median age of 65 years. Chronic HCV infection was the main underlying disease process, and half the patients included were BCLC stage $\mathrm{C}$ at the time of diagnosis. The median OS was 7.5 weeks, and the best outcomes were partial response in $11.8 \%$ of patients and stable disease in $23.5 \%$ of patients. Approximately $35.3 \%$ at the first investigation on imaging at $8-12$ weeks. Fifty-eight patients had died at the time of data analysis, with the most common causes of death attributed to disease progression, acute liver failure and variceal bleeding. During the treatment, $5.9 \%$ of patients showed grade 3 TRAE including bullous lichenoid drug eruption and hepatitis. Also, the multivariate analysis showed Child-Pugh stage was an independent risk factor for survival. In conclusion, the study confirmed the safety of nivolumab therapy in patients with advanced HCC but despite this therapy, prognosis of patients with advanced cirrhosis remained poor, and the exact benefit of nivolumab required further research (12).

\section{CheckMate-459}

CheckMate 459 was a phase III, randomized multicenter study that evaluated the clinical efficiency and safety of nivolumab vs. sorafenib as a first line treatment in patients with unresectable disease. Patients included had no prior exposure to chemotherapy with advanced HCC confirmed pathologically, either not eligible for surgical or locoregional therapies or had progression after surgical and/or locoregional therapy. Patient also had Child-Pugh class A cirrhosis, and ECOG score of 0 or 1 . Patients were randomized 1:1 to either IV nivolumab $240 \mathrm{mg}$ every 2 weeks or PO sorafenib $400 \mathrm{mg}$ twice a day. Primary endpoint was OS, with secondary endpoints consisting of ORR, PD-L1 expression and progressive-free survival.

Seven hundred and forty-three patients were involved in the study, randomized into one of two groups: 371 participants in the nivolumab group and 372 participants in the sorafenib group, with minimum follow up of 22.8 months. Patients were treated until disease progression or unacceptable toxicity. Median OS was 16.4 months for nivolumab group and 14.7 months for sorafenib group (HR 0.85; $\mathrm{P}=0.0752$ ), which was not statistically significant. Objective response rate was $15 \%$ and $7 \%$, for nivolumab and sorafenib respectively. High grade TRAE were reported in $22 \%$ of patients in the nivolumab group, and $49 \%$ in the sorafenib group, and led to treatment termination in $4 \%$ and $8 \%$ of patients respectively. Though not statistically significant, nivolumab showed clinically significant improvement in OS, objective response rate and complete response, and showed a favorable safety profile (13). In conclusion, nivolumab showed two-fold higher objective response rate, more complete responses, less severe adverse events, and a lower rate of discontinuing therapy because of side effects.

\section{Pembrolizumab}

Pembrolizumab is a popular human IgG4 monoclonal antibody to PD-1 which has shown improvement in OS in non-small cell lung and breast cancer. Several studies have been conducted to solidify pembrolizumab as a secondchoice therapy of advanced HCC. It was also granted accelerated FDA approval in patient with HCC who had been formerly treated with sorafenib, based on the KEYNOTE-224 trial (14).

\section{KEYNOTE-224}

KEYNOTE-224 was a phase II, non-randomized, open label trial that assessed efficacy and safety of pembrolizumab in advanced HCC patients who progressed on sorafenib or intolerability. Patients involved in this trial had confirmed advanced HCC, Child-Pugh class A cirrhosis, and ECOG status $0-1$. Patients were administered IV pembrolizumab every 3 weeks for approximately 2 years or until disease advancement, intolerable toxicity, or voluntary treatment discontinuation. Primary endpoint was ORR, described as the ratio of participants with complete or partial response in all patients who received at least one dose of pembrolizumab. Safety was the secondary endpoint.

After screening 194 patients, 104 were found to be eligible. These patients also had BCLC stage B or C that was not eligible or was progressive after locoregional therapy or refractory to curative treatment approaches and predicted life expectancy greater than 3 months. Patients were treated until disease progression, intolerable toxicity, or 2 years. The objective response was $17 \%$, with the best overall responses being complete (1\%) and partial (16\%) responses. Patients with stable disease comprised $73 \%$ of eligible patients and $33 \%$ had disease progression. Grade 3 TRAE occurred in $24 \%$ of patients, with the most common being increased AST, increased ALT, and fatigue. Grade 4 TRAE of increased bilirubin occurred in $1 \%$ of patients, and one death occurred that was associated with ulcerative esophagitis. This study showed that pembrolizumab had a tolerable profile in patients with advanced HCC, and due 
to its effectiveness, could be a possible treatment option for these patients (15).

\section{KEYNOTE-240}

KEYNOTE-240 was a phase III, double-blind study which compared the efficacy and safety of pembrolizumab plus standard supportive care versus placebo plus standard care in patients with HCC previously treated with sorafenib. Participants had histologically or cytologically confirmed diagnosis of advanced HCC with progression on radiological studies after treatment with sorafenib or intolerance to sorafenib, Child-Pugh Stage A, ECOG performance status 0 or 1 and life expectancy of more than 3 months, which reflected the demographics of patients in KEYNOTE-224 trial. Patients were assigned to two arms: IV pembrolizumab $200 \mathrm{mg}$ every 3 weeks and best supportive care or placebo every 3 weeks plus best supportive care for approximately 35 cycles, until disease progression or intolerable toxicity. Best supportive care was defined as pain control and management of potential complications such as ascites according to local standards of care. Primary endpoints included progressive free survival and OS. Secondary endpoints included ORR, duration of response, disease control rate, time to progression and evaluation of safety and tolerability.

Four-hundred and fourteen participants were enrolled in this study, with inclusion criteria as followed: confirmed diagnosis of advanced HCC, BCLC Stage C disease or Stage B not appropriate for or was refractory to locoregional therapy or curative treatment approach, ECOG performance status 0 or 1 , chronic HCV status treated and untreated, and controlled HBV infection. Patients were randomized into 2 arms: 278 patients received IV pembrolizumab $200 \mathrm{mg}$ every three weeks plus supportive care and 135 patients received IV $0.09 \%$ normal saline every three weeks plus supportive care for 35 cycles or less. Pembrolizumab enhanced OS (HR 0.78; $\mathrm{P}=0.023$ ) and improved progressive free survival (HR 0.78; $\mathrm{P}=0.020$ ) when compared to placebo, but results were found to be statistically not significant based on predetermined criteria. Objective response rate was $16.9 \%$ for pembrolizumab versus $2.2 \%$ for placebo. Grade 3-4 TRAE were occurred in $60.9 \%$ of pembrolizumab group versus $48.5 \%$ of placebo group, with the most common being AST elevation, hyperbilirubinemia, fatigue and pruritis. One death contributed to treatment occurred in the pembrolizumab group, secondary to malignant neoplasm progression. Without meeting statistical significance, pembrolizumab was shown to increase OS and PFS in patients with advanced HCC previously treated with sorafenib when compared to placebo, reducing the risk of death by $22 \%$. The safety profile was also found to be consistent with KEYNOTE-224 findings and showed further evidence for the use of pembrolizumab as second line therapy in advanced HCC patients (16).

\section{Durvalumab}

Durvalumab is another human monoclonal which has been researched in the management of advanced HCC. A phase I/II, multicenter open-label clinical trial which evaluated efficacy and safety of durvalumab as monotherapy in patients with solid tumors in late stages, including HCC conducted by international researchers. Patients were given durvalumab every 14 days for approximately 1 year, or until disease progression. The primary endpoint was evaluation of safety profile while secondary endpoint was antitumor activity measured by the RECIST assessment. One thousand and twenty-two patients were enrolled who had histologically confirmed advanced solid tumors that were either not responsive to standard therapy or with no standard therapy at all, ECOG status $0-1$, and able to provide a tissue biopsy. Particularly, 40 patients were included who had HCC (Child-Pugh stage A) with $93 \%$ having received sorafenib in the past. TRAE occurred in $80 \%$ of the patients including fatigue, pruritis and elevated AST. Grade 3-4 TRAE occurred in 20\% of the patients that included transaminitis. Antitumor activity was measured in many ways, including OS rate at 12 months was $56 \%$, with greater OS rate of $83 \%$ in $\mathrm{HCV}$ positive patients. The study results showed that durvalumab had a tolerable safety profile and showed favorable improvement in antitumor activity and OS in HCC patients with chronic HCV (17).

\section{CTLA-4 Inhibitors}

Cytotoxic T-lymphocyte antigen 4 (CTLA-4) that is expressed on activated $\mathrm{T}$ cells and $\mathrm{T}$ regulator cells, is another target for immune checkpoint inhibition that has been explored as immunotherapy for HCC. Binding of CTLA-4 and PD-1/PD-L1 to ligands in the tumor cell leads to $\mathrm{T}$ lymphocyte weakening, which allows the malignant cells to escape elimination by host immune cells. Under physiological conditions without treatment with ICPIs, T cells are activated by cancer antigens presented by antigen-presenting dendritic cells (DC) in the lymphatic 
tissue of the tumor beds. CTLA-4 causes production of an inhibitory signal that competes with stimulus by CD28 molecule when ligated by B7 on the DC. CTLA-4 also augments the repressive function of $\mathrm{T}$ regulator cells. Activated T-cells then travel to sites where the recognition of oncologic antigens increases production of PDL-1 on the tumor cells (18). Disruption/inhibition of CTLA-4 interaction leads to tumor rejection through enrichment of $\mathrm{T}$ cell-effector responses and is associated with selective T-regulatory cell depletion (19). The most promising CTLA-4 inhibitor under current research for advanced HCC is tremelimumab.

\section{Tremelimumab}

Tremelimumab is a human IgG2 monoclonal antibody that inhibits binding of CTLA-4 which stimulates responses in a subset of patients who have metastatic melanoma and colorectal cancer (20). This CTLA-4 inhibitor along with ipilimumab were ground-breaking in the field of stimulatory monoclonal antibodies for cancer therapy and opened the door for clinical development of other targets on immune cells for the treatment of various malignancies (21).

\section{NCT01008358: CTLA-4 blockade with tremelimumab trial}

This phase II, open label, multicenter clinical trial was designed to test antineoplastic activity of tremelimumab in patients with HCC with chronic HCV infection, and its safety profile in patients with cirrhosis. Patients eligible for this study had advanced HCC that was unresectable and not amenable to locoregional treatment, at least 4 weeks without sorafenib or any systemic treatment, ECOG score less than 2, Child-Pugh stage A or B, and life expectancy of more than 3 months. Patients were treated with IV tremelimumab every 90 days until tumor progression or intolerability. The primary endpoints were OR and disease control rate, while secondary endpoint was change in $\mathrm{HCV}$ viral load.

Twenty-one patients were able to be assessed for toxicity and viral load changes and 17 patients were assessable for tumor response. Of these patients, $43 \%$ had advanced at least Child-Pugh stage B with altered liver function, and $76 \%$ were naive to sorafenib. Patients were treated with IV tremelimumab 15 milligrams per kilogram every 3 months until either tumor advancement or toxicity. No complete responses were observed, and partial responses in $17.6 \%$ of patients assessable for tumor response. Disease control rate was $76 \%$ and median duration to progression was 6.48 months. Results also found a median OS rate of 8.2 months. Tremelimumab decreased the HCV viral load with median values of $30,000 \mathrm{IU} / \mathrm{mL}$ at day 120 and $1,690 \mathrm{IU} / \mathrm{mL}$ at day 210. The most common TRAE consisted of fatigue, skin rash, and elevated AST and ALT. Overall, the study showed a tolerable safety profile with no treatment related mortality, antitumor and antiviral activity that warranted more investigation into treatment of advanced HCC in patients with chronic HCV (22).

\section{Combination of Two Checkpoint Inhibitors}

Based on current ongoing research, ICPIs against PD-1/ PD-L1 and CTLA-4 have shown promising results in the treatment of non-resectable HCC. Due to these findings, there has been a surge in trials evaluating the simultaneous blockade of several immune checkpoints, which has shown high efficacy in the treatment of other solid neoplasms. It is theorized that the inhibition of PD-1/PD-L1 pathway alone may not activate tumor immunity as expected if there are not sufficient amounts of $\mathrm{CD} 8^{+}$cells in the tumor microenvironment, but with simultaneous inhibition of B7CTLA-4 pathway, there may be an increase in abundance of $\mathrm{CD}^{+}$cells in the lymph nodes, which leads to an increase in activated $\mathrm{CD} 8^{+}$cells invading the tumor, which enhances their antitumor effects (2). Due to this theory, the combination of ICPI as treatment in advanced HCC is worth further investigation.

\section{Nivolumab plus Ipilimumab: partial cobort of CheckMate 040}

Based on CheckMate 040, nivolumab monotherapy was approved for patient with HCC previously treated with sorafenib. The combination of nivolumab and ipilimumab has shown positive results in treatment of malignant melanoma, including improvement in ORR and in median progressive free survival in combination group in CheckMate 067, with $58.9 \%$ and 11.7 months respectively (23). A sub-cohort of CheckMate 040 trial patients were involved in a study evaluating the efficacy and safety of nivolumab and ipilimumab in patients with late-stage HCC who had been previously treated with sorafenib. Patients involved in this study were eligible if they had histologically confirmed advanced HCC either not amenable for surgical and/or radiation therapies or had progression on these therapies, ECOG score of 0 or 1 , and Child-Pugh score of 6 or less. Patients were allocated into 3 
arms: Group 1: nivolumab + ipilimumab every 3 weeks for 4 doses, Group 2: nivolumab + ipilimumab every 3 weeks for 4 doses followed by nivolumab every 2 weeks or Group 3: nivolumab + ipilimumab every 6 weeks. The primary endpoints were safety and ability to tolerate, with secondary endpoints being ORR, duration of treatment, OS, and disease control rate.

One hundred and forty-eight patients were randomized into 3 groups. Most of the patients had baseline vascular invasion, had BCLC stage $\mathrm{C}$ or had discontinued sorafenib due to disease progression. Patients were followed for 24 months at a minimum or until intolerable toxicity or disease progression. Objective response rate was equivalent (15-16\%) across all three groups with 4 patients achieving complete response in G1, and 3 pts in G2. Patients in G1 showed median OS of 23 months, and the highest OS rate at 24 months of $48 \%$. The combination of nivolumab + ipilimumab tolerated well with $37 \%$ of patients experiencing high grade treatment related adverse events; the most common being pruritis and rash. In conclusion, combination of nivolumab + ipilimumab had an objective response rate double that of nivolumab monotherapy in CheckMate 040 (31-32\% vs. 14\% respectively) and had a tolerable safety profile. Most promising of all results was median OS in G1 with 23 months (24).

\section{NCT02519348: Durvalumab and tremelimumab}

This phase I/II, open-label, randomized study set designed to assess safety of durvalumab/tremelimumab combination in patients with advanced HCC. Patients eligible for this study had confirmed HCC based on histology, had no exposure to prior immunotherapy, and had progressed or were intolerant to sorafenib. In this study, patients with or without chronic $\mathrm{HBV}$ or $\mathrm{HCV}$ infection were given a regimen of a combination durvalumab and tremelimumab followed by durvalumab alone. The primary endpoints included the number of patients who reported serious adverse events and those experiencing dose limiting effects. Subsequent endpoints included ORR, disease control rate, and OS.

As of January 2017, 40 subjects were involved in this study, with $11 \mathrm{HBV}+$ patients, $9 \mathrm{HCV}+$ patients and 20 patients that were uninfected. Other characteristics of this cohort included $93 \%$ of patients with Stage A cirrhosis and $30 \%$ of patients with no prior exposure to systemic therapy. Patients were given a regimen of combination of durva/ tremelim (IV $20 \mathrm{mg} / \mathrm{kg}$ and $1 \mathrm{mg} / \mathrm{kg}$ respectively) every
4 weeks for 4 doses followed by IV durvalumab every 4 weeks for approximately 16 weeks. Grade 1 or higher TRAE were found in $60 \%$ of patients, with most common being fatigue, increased ALT and pruritis. The most common Grade 3 or above TRAE was asymptomatic elevation of AST in $10 \%$ of patients. Sixty percent of patients had to discontinue treatment due to various reasons: 16 patients due to disease progression, 3 patients due to serious TRAE (grade 4 transaminitis, grade 3 pneumonitis and colitis/diarrhea) and 4 patients due to non-treatment related death such as cardiac arrest and esophageal variceal bleeding. Objective response rate defined as partial response was shown in $30 \%$ of uninfected patients, and there was an overall disease control rate at 16 weeks of $57 \%$ across all patients. In conclusion, there were no unexpected treatment related adverse effects, and antitumor activity was predominantly observed in $\mathrm{HBV} / \mathrm{HCV}$ - patients. The limitation of this study was a small sample size, and enrollment is ongoing (25).

\section{Ongoing and future studies}

Sorafenib has been cleared for usage in treatment of advanced HCC since 2007, however reported response rates remain poor in current clinical trials, ranging from $2.3 \%$ to $9.2 \%$, leaving an urgent need for more tolerable and effective options for treatment (26). Several clinical trials have shown the advantages of ICPI as treatment for advanced HCC, showcased by the FDA approval of nivolumab for first line treatment of those previously treated or progressed on sorafenib. This has opened the door for a surge of clinical trials with different ICPIs, combinations and neo-adjuvant therapy as treatment for ICPI.

Several studies have been performed on the participants involved in the CheckMate 040 trial. FRI-499 is a study utilizing the cohort of patients with Child-Pugh B disease and advanced HCC from CheckMate 040 to study the efficacy and hepatic safety profile in these patients. Patients with Child-Pugh B (CP-B) were divided into 2 groups: treated with or not treated with, who received IV nivolumab $240 \mathrm{mg}$ for every 14 days until unacceptable toxicity or disease progression. These patients had to be free of hepatic encephalopathy or paracentesis within 6 months or within 3 months of screening respectively. The primary outcome was ORR, and secondary was safety. Twenty-five sorafenib naïve and 24 sorafenib exposed patients comprised this cohort, with $59 \%$ of patients having $\mathrm{HBV}$ or $\mathrm{HCV}$ infection, and $39 \%$ of the patients with alpha-fetoprotein (AFP) levels 
above $400 \mathrm{ug} / \mathrm{L}$. Objective response rate was $10 \%$, and median duration of response was 9.9 months. Four out of 5 responders saw an improvement of Child-Pugh status to A5 or A6 which was sustained for 6 months. Serious TRAE occurred in $2 \%$ of patients, leading to discontinuation, and patients with AFP levels less than $400 \mathrm{ug} / \mathrm{L}$ had stable disease $(57 \%)$ and higher disease control rate (68\%) than patients with baseline AFP levels above 400. Results of this study demonstrated manageable safety and tolerability of nivolumab in patients with advanced HCC and Child-Pugh B disease. Future results will compare data with patients with $\mathrm{CP}-\mathrm{A}$, advanced $\mathrm{HCC}$, and $\mathrm{HBV} / \mathrm{HCV}$ viral kinetics and extended follow up in this cohort (27).

Despite advancements in the treatment of early HCC, curative options remain associated with high rates of recurrence at approximately $70 \%$ at 5 years. The use of adjuvant therapy may reduce this amount of recurrence. There is a strong argument for combined therapy of immunotherapy and surgery and/or locoregional treatment because of the treatment-induced reduction of tumorassociated antigens seen in other solid tumors (28). Amongst the several trials of HCC therapy designed to assess the safety and efficacy of adjuvant treatment in patient at significant recurrence risk after resection or ablation. There also is a study currently enrolling to test nivolumab against placebo in the adjuvant following hepatic surgical excision or local ablation (NCT03383458). KEYNOTE-937 is a trial with pembrolizumab as neo-adjuvant therapy is currently ongoing, and there are phase II trials being conducted for tremelimumab in the similar setting (2). The implementation of ICPI as neo- and adjuvant therapy could change the recurrence rate for HCC patients treated with chemoembolization and trans-arterial radioembolization.

\section{Conclusions}

The prognosis continues to be bleak for HCC, especially in the advanced stages. Previous studies regarding advanced HCC and shown response rates of $2-3 \%$ and for sorafenib and 7\% for regorafenib\% (29). And despite expanded options including lenvatinib, cabozantinib and ramucirumab, estimated survival remains less than one year, causing the necessity for new therapies. ICPI have been investigated for several tumor types to establish an effective alternative treatment option to previously approved treatments for this malignancy. These medications induce the immune system to generate an anti-neoplastic response by blocking the interaction of PD-L1/PD-1 ligand/ receptor pair. The best responses have been demonstrated in malignancies with high mutation burdens such as lung cancers and melanoma $(30,31)$.

Several studies of monotherapy and combination ICPI therapy in advanced HCC patients who were previously treated with sorafenib have shown some improvement in PFS and ORR when compared to current first line therapy. But these findings should be taken with caution, as the same has not been found in the clinical setting. The use of ICPIs in advanced HCC may still fail to accomplish the treatment requirements, due to $30-40 \%$ of failing to respond to these treatments in real-life clinical practice. The eligibility rate for ICPIs is close to $10-20 \%$ in the first line, and less than $10 \%$ in the second line. The use of a combination of ICPIs may overcome the primary resistance to immunotherapy. Use of double or triple combinations of immunotherapies associated with immune checkpoint inhibition may show the most promising results (2).

It is also necessary to identify available biomarkers that can predict tumor response, prognosis, and help identify subgroups of patients with HCC who will respond best to immunotherapy. Based on the literature, circulating amounts of PD-L1 may be the answer. Some metanalyses performed found that higher PD-L1 concentrations predicted poor differentiation of tumors, higher levels of AFP, invasion into the blood vessels, and worse survival rates in patients with HCC $(32,33)$. A research study performed in 2016 sought to investigate the prognostic value of soluble PD-L1 (sPD-L1) levels in patients with HCC and found promising results. It showed sPD-L1 levels were positively correlated with both cirrhosis and HCC stages, and that levels associated positively with sCD163, a marker of macrophage activation, and CRP. Also, patients with elevated serum concentrations of sPD-L1 had increased mortality risk (HR 3.340, $\mathrm{P}<0.001$ ) while those with minimal concentration seemed to have better prognoses, and elevated levels of sPD-L1 was associated with increased mortality independent of AFP and sCD163 levels and stage of cirrhosis (34).

In conclusion, it is quite evident that immunotherapy with immune blocking agents such as nivolumab, pembrolizumab and other ICPIs may have a promising future in the treatment of advanced HCC due to recent studies, but there is a need for correlation of these results in the current clinical climate. Prevention of certain causes such as vaccination initiatives at birth for HBV may help curb the prevalence of chronic hepatitis B infection, and effective antiviral treatments for $\mathrm{HCV}$ may cure hepatitis related to the virus. At this time, there are lower relative 
risks of HCC development in cirrhosis due to obesity and diabetes when compared to viral hepatitis but based on the high prevalence of metabolic syndrome in the Western parts of the world, it is anticipated that these factors may become more common causes of HCC in future decades. Because of the current data for immunotherapy for advanced HCC, the need for real world results is much needed, and may lie in the discovery of easily accessible biomarkers to predict a cohort of the HCC population for treatment with the highest chance for remission or poorest prognosis. With these additional factors, the use of ICPI can provide effective outcomes for those suffering from advanced HCC.

\section{Acknowledgments}

Funding: None.

\section{Footnote}

Reporting Checklist: The authors have completed the Narrative Review reporting checklist. Available at https:// dx.doi.org/10.21037/sci-2021-029

Conflicts of Interest: All authors have completed the ICMJE uniform disclosure form (available at https://dx.doi. org/10.21037/sci-2021-029). The authors have no conflicts of interest to declare.

Ethical Statement: The authors are accountable for all aspects of the work in ensuring that questions related to the accuracy or integrity of any part of the work are appropriately investigated and resolved.

Open Access Statement: This is an Open Access article distributed in accordance with the Creative Commons Attribution-NonCommercial-NoDerivs 4.0 International License (CC BY-NC-ND 4.0), which permits the noncommercial replication and distribution of the article with the strict proviso that no changes or edits are made and the original work is properly cited (including links to both the formal publication through the relevant DOI and the license). See: https://creativecommons.org/licenses/by-nc-nd/4.0/.

\section{References}

1. SEER Cancer Stat Facts: Liver and Intrahepatic Bile Duct Cancer. National Cancer Institute. Bethesda, MD, USA. Available online: https://seer.cancer.gov/statfacts/html/ livibd.html

2. Giannini EG, Aglitti A, Borzio M, et al. Overview of Immune Checkpoint Inhibitors Therapy for Hepatocellular Carcinoma, and The ITA.LI.CA Cohort Derived Estimate of Amenability Rate to Immune Checkpoint Inhibitors in Clinical Practice. Cancers (Basel) 2019;11:1689.

3. Ghouri YA, Mian I, Rowe JH. Review of hepatocellular carcinoma: Epidemiology, etiology, and carcinogenesis. J Carcinog 2017;16:1.

4. Li D, Sedano S, Allen R, et al. Current Treatment Landscape for Advanced Hepatocellular Carcinoma: Patient Outcomes and the Impact on Quality of Life. Cancers (Basel) 2019;11:841.

5. Llovet JM, Ricci S, Mazzaferro V, et al. Sorafenib in advanced hepatocellular carcinoma. $\mathrm{N}$ Engl J Med 2008;359:378-90.

6. Kudo M, Finn RS, Qin S, et al. Lenvatinib versus sorafenib in first-line treatment of patients with unresectable hepatocellular carcinoma: a randomised phase 3 noninferiority trial. Lancet 2018;391:1163-73.

7. Kelley RK. Atezolizumab plus Bevacizumab - A Landmark in Liver Cancer. N Engl J Med 2020;382:1953-5.

8. Finn RS, Qin S, Ikeda M, et al. Atezolizumab plus Bevacizumab in Unresectable Hepatocellular Carcinoma. N Engl J Med 2020;382:1894-905.

9. Galle PR, Finn RS, Qin S, et al. Patient-reported outcomes from the phase III IMbrave150 trial of atezolizumab (atezo) + bevacizumab (bev) versus sorafenib (sor) as first-line treatment (tx) for patients with unresectable hepatocellular carcinoma (HCC). J Clin Oncol 2020;38;476.

10. Keenan BP, Fong L, Kelley RK. Immunotherapy in hepatocellular carcinoma: the complex interface between inflammation, fibrosis, and the immune response. J Immunother Cancer 2019;7:267.

11. Francisco LM, Salinas VH, Brown KE, et al. PD-L1 regulates the development, maintenance, and function of induced regulatory T cells. J Exp Med 2009;206:3015-29.

12. Umemoto Y, Okano S, Matsumoto Y, et al. Prognostic impact of programmed cell death 1 ligand 1 expression in human leukocyte antigen class I-positive hepatocellular carcinoma after curative hepatectomy. J Gastroenterol 2015;50:65-75.

13. El-Khoueiry AB, Sangro B, Yau T, et al. Nivolumab in patients with advanced hepatocellular carcinoma (CheckMate 040): an open-label, non-comparative, phase 1/2 dose escalation and expansion trial. Lancet 2017;389:2492-502. 
14. Finkelmeier F, Czauderna C, Perkhofer L, et al. Feasibility and safety of nivolumab in advanced hepatocellular carcinoma: real-life experience from three German centers. J Cancer Res Clin Oncol 2019;145:253-9.

15. Sangro B, Park JW, Dela Cruz CM, et al. A randomized, multicenter, phase 3 study of nivolumab vs. sorafenib as first-line treatment in patients with advanced hepatocellular carcinoma (HCC): CheckMate 459. J Clin Oncol 2016. doi: 10.1200/JCO.2016.34.15_suppl. TPS4147.

16. El Dika I, Khalil DN, Abou-Alfa GK. Immune checkpoint inhibitors for hepatocellular carcinoma. Cancer 2019;125:3312-9.

17. Zhu AX, Finn RS, Edeline J, et al. Pembrolizumab in patients with advanced hepatocellular carcinoma previously treated with sorafenib (KEYNOTE-224): a non-randomised, open-label phase 2 trial. Lancet Oncol 2018;19:940-52.

18. Finn RS, Ryoo BY, Merle P, et al. Pembrolizumab As Second-Line Therapy in Patients With Advanced Hepatocellular Carcinoma in KEYNOTE-240: A Randomized, Double-Blind, Phase III Trial. J Clin Oncol 2020;38:193-202.

19. Wainberg ZA, Segal NH, Lee KH, et al. Safety and clinical activity of durvalumab monotherapy in patients with hepatocellular carcinoma (HCC). J Clin Oncol 2017;35:4071.

20. Cheng H, Sun G, Chen H, et al. Trends in the treatment of advanced hepatocellular carcinoma: immune checkpoint blockade immunotherapy and related combination therapies. Am J Cancer Res 2019;9:1536-45.

21. Lleo A, Rimassa L, Colombo M. Hepatotoxicity of immune check point inhibitors: Approach and management. Dig Liver Dis 2019;51:1074-8.

22. Flynn MJ, Sayed AA, Sharma R, et al. Challenges and Opportunities in the Clinical Development of Immune Checkpoint Inhibitors for Hepatocellular Carcinoma. Hepatology 2019;69:2258-70.

23. Sangro B, Gomez-Martin C, de la Mata M, et al. A clinical trial of CTLA-4 blockade with tremelimumab in patients with hepatocellular carcinoma and chronic hepatitis C. J

doi: $10.21037 /$ sci-2021-029

Cite this article as: Biglow L, Ashraf S, Alsharedi M. Checkpoint inhibitors: literature review of new treatments for hepatocellular carcinoma. Stem Cell Investig 2021;8:22.
Hepatol 2013;59:81-8.

24. Silva IP, Long GV. Systemic therapy in advanced melanoma: integrating targeted therapy and immunotherapy into clinical practice. Curr Opin Oncol 2017;29:484-92.

25. Yau T, Kang YK, Kim TY, et al. Nivolumab (NIVO) + ipilimumab (IPI) combination therapy in patients with advanced hepatocellular carcinoma: results from CheckMate 040. J Clin Oncol 2019;37:4012.

26. Kelley RK, Abou-Alfa GK, Bendell JC, et al. Phase I/II study of durvalumab and tremelimumab monotherapy in patients with hepatocellular carcinoma (HCC): Phase I safety and efficacy analyses. J Clin Oncol 2017;35:4073.

27. Liu X, Qin S. Immune Checkpoint Inhibitors in Hepatocellular Carcinoma: Opportunities and Challenges. Oncologist 2019;24:S3-S10.

28. Sangro, B, Matilla A, Santoro A, et al. FRI-499-Efficacy and hepatic safety of nivolumab treatment in patients with Child-Pugh B disease and advanced hepatocellular carcinoma in CheckMate 040. J Hepatol 2019;70:e619.

29. Bruix J, Qin S, Merle P, et al. Regorafenib for patients with hepatocellular carcinoma who progressed on sorafenib treatment (RESORCE): a randomised, double-blind, placebo-controlled, phase 3 trial. Lancet 2017;389:56-66.

30. Weber J, Mandala M, Del Vecchio M, et al. Adjuvant Nivolumab versus Ipilimumab in Resected Stage III or IV Melanoma. N Engl J Med 2017;377:1824-35.

31. Borghaei H, Paz-Ares L, Horn L, et al. Nivolumab versus Docetaxel in Advanced Nonsquamous Non-Small-Cell Lung Cancer. N Engl J Med 2015;373:1627-39.

32. Robert C, Long GV, Brady B, et al. Nivolumab in previously untreated melanoma without BRAF mutation. N Engl J Med 2015;372:320-30.

33. Gu X, Gao XS, Xiong W, et al. Increased programmed death ligand-1 expression predicts poor prognosis in hepatocellular carcinoma patients. Onco Targets Ther 2016;9:4805-13.

34. Finkelmeier F, Canli Ö, Tal A, et al. High levels of the soluble programmed death-ligand (sPD-L1) identify hepatocellular carcinoma patients with a poor prognosis. Eur J Cancer 2016;59:152-9. 